\title{
Evolución de la textura durante el recocido continuo de un acero laminado en frío
}

\author{
Ma M. PETITE, I. GUTIÉRREZ \\ CEIT y ESII (Univ. Navarra), Po Manuel Lardizabal 15, 20009 Donostia-San Sebastián
}

\begin{abstract}
En el presente trabajo, se ha realizado un análisis de la evolución de la textura de recristalización desarrollada en un acero deformado en frío, con un contenido ultrabajo en carbono, $0.0033 \%$, al ser recocido a diferentes temperaturas. Los cambios observados se han contrastado con los dos mecanismos propuestos en bibliografía para interpretar la formación de las texturas de recristalización: nucleación orientada y crecimiento selectivo.
\end{abstract}

Palabras clave: Acero ultra bajo en carbono, recocido continuo, recristalización, textura.

\section{Texture evolution in cold-rolled steel during continuous annealing}

In the present work the recrystallization texture evolution in a cold rolled ultra-low carbon steel, $0.0033 \%$, after annealing at different temperatures has been analysed. The results have been discused in terms of the mechanisms reported in the literature to explain the recrystalization texture development, oriented nucleation and selective growth.

Keywords: Ultra-low carbon steel, continuous annealing, recrystallisation, texture.

\section{INTRODUCCIÓN}

La capacidad de embutición de un material y la uniformidad de propiedades en las diferentes direcciones sobre el plano de laminación, que es lo que evita la formación de orejas al conformarlo, están directamente relacionadas con la textura. La textura final de un material depende, en general, de las condiciones de procesamiento. En el caso concreto de los aceros laminados en frío y recocidos en continuo, la composición química junto con la etapa de laminación en caliente, la propia reducción en frío y los parámetros empleados en el ciclo de recocido definen la textura final del material.

La textura de los aceros queda generalmente definida mediante una Fibra $\gamma$ completa y una Fibra $\alpha$ parcial. La Fibra $\gamma$ corresponde a las orientaciones de los granos que constituyen el acero para las cuales los planos $\{111\}$ de la red cristalina de la ferrita coinciden con el plano de laminación. La Fibra $\alpha$ corresponde a orientaciones en las que la dirección $<110>$ de la citada red, en los diferentes granos de ferrita, es paralela a la dirección de laminación (1). Una textura óptima para la embutición en una chapa de acero es aquella que posee una Fibra $\gamma$ intensa, en detrimento de orientaciones pertenecientes a la fibra $\alpha \mathrm{y}$ a todas aquellas en las que el plano $\{100\}$ de la ferrita coincide con el de laminación.

Entender los cambios que tienen lugar en la textura del material tras la aplicación del tratamiento de recocido es un requisito esencial a la hora de definir las óptimas condiciones del ciclo de recocido continuo que proporcionen la mejor textura de cara a un proceso posterior de embutición.

\section{PROCEDIMIENTO EXPERIMENTAL}

El material estudiado es un acero comercial ultrabajo en carbono y calmado con aluminio producido por ACERALIA S.A. Su composición se muestra en la Tabla I. El acero se ha
TABLA I. COMPOSICIÓN OUÍMICA DEL ACERO.

\begin{tabular}{llllll}
\hline$\% \mathbf{C}$ & $\% \mathbf{M n}$ & $\% \mathbf{S i}$ & $\% \mathbf{P}$ & $\% \mathbf{A l}$ & $\% \mathbf{N}$ \\
\hline 0.0033 & 0.29 & 0.007 & 0.011 & 0.029 & 0.0031 \\
\hline
\end{tabular}

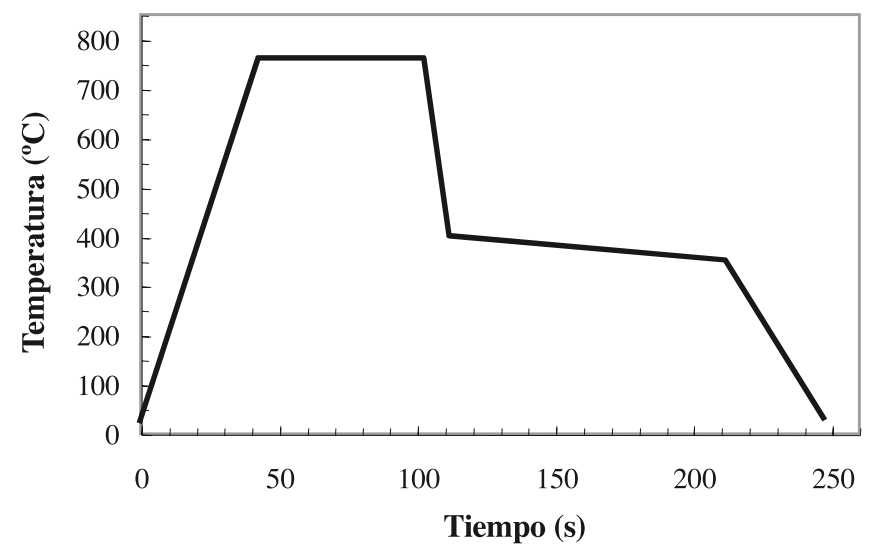

Figura 1. Ciclo de recocido continuo.

suministrado en la condición de deformado en frío, con una reducción total del $86.9 \%$.

Los diferentes ciclos de recocido se han realizado utilizando un simulador de recocido continuo (2) que permite una reproducción fiable de los ciclos industriales a nivel de laboratorio. En la Fig. 1 se muestra un ejemplo de un perfil de los ciclos simulados. En el presente trabajo se han variado las temperaturas de recocido entre 450 y $800^{\circ} \mathrm{C}$, manteniendo fijos el resto de los parámetros del ciclo de recocido, con el fin de obtener una amplia gama de condiciones microestructurales. 


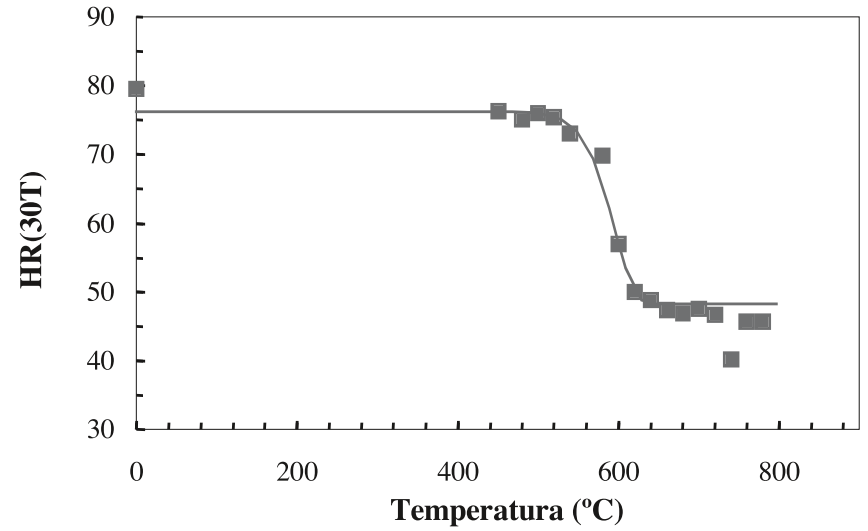

Figura 2. Evolución de la dureza con la temperatura de recocido.

Las medidas de textura se han obtenido en un difractómetro de rayos- $X$ PHILLIPS, modelo $X^{\prime}$ Pert-MRD, con radiación Co $\left(\mathrm{K}_{\alpha}\right)$. La función de distribución de orientaciones, FDO, se ha calculado a partir de cuatro figuras de polos incompletas $\{110\},\{200\},\{112\}$ y $\{310\}$. Los resultados finales de las FDO se han obtenido mediante el programa MTM-FHM desarrollado por Van Houte (3).

\section{RESULTADOS Y DISCUSIÓN}

Una de las propiedades mecánicas que varia con la temperatura de recocido es la dureza. La disminución provocada en dicha propiedad al variar la temperatura de recocido está directamente relacionada con la recristalización del material (4). En la Fig. 2 se muestra la curva de dureza obtenida para el presente acero en función de la temperatura de mantenimiento del ciclo de recocido. El importante ablandamiento observado en el rango de temperaturas $520-640^{\circ} \mathrm{C}$ coincide precisamente con la recristalización sufrida por el material, tal como se desprende de las micrografías de la Fig. 3.

La textura del material en las condiciones de deformación en frío se muestra mediante la sección $\varphi_{2}=45$ de la FDO en la Fig. 4. En la citada figura se puede observar la presencia de una Fibra $\gamma$ bastante homogénea en todas sus componentes. Asimismo, se aprecia una mayor intensidad para la Fibra $\alpha$ parcial junto con una alta densidad para las orientaciones próximas a $\{100\}<110>$.

El recocido del material deformado en frío, a través de los cambios microestructurales que induce en el acero, recristalización, crecimiento de grano, modifica la textura de partida. El conseguir una buena embutibilidad depende, entre otros muchos factores, de las condiciones empleadas durante el recocido.

En las Figs. 5 y 6 se muestra el desarrollo seguido por ambas fibras al variar la temperatura del ciclo de recocido. Inicialmente, en el rango de temperaturas de recocido más bajas, comprendido entre 480 y $600^{\circ} \mathrm{C}$ la textura del material deformado no experimenta modificaciones importantes a pesar del inicio de la recristalización. Así para el caso de la temperatura de $600^{\circ} \mathrm{C}$, en la que a partir de medidas metalográficas se determinó una fracción recristalizada del $46 \%$, las densidades obtenidas en las dos fibras, $\gamma$ y $\alpha$, están muy próximas a las observadas en el estado deformado. El mantenimiento de la textura del estado deformado con el inicio de la recristalización puede ser explicado a partir de las argumen-
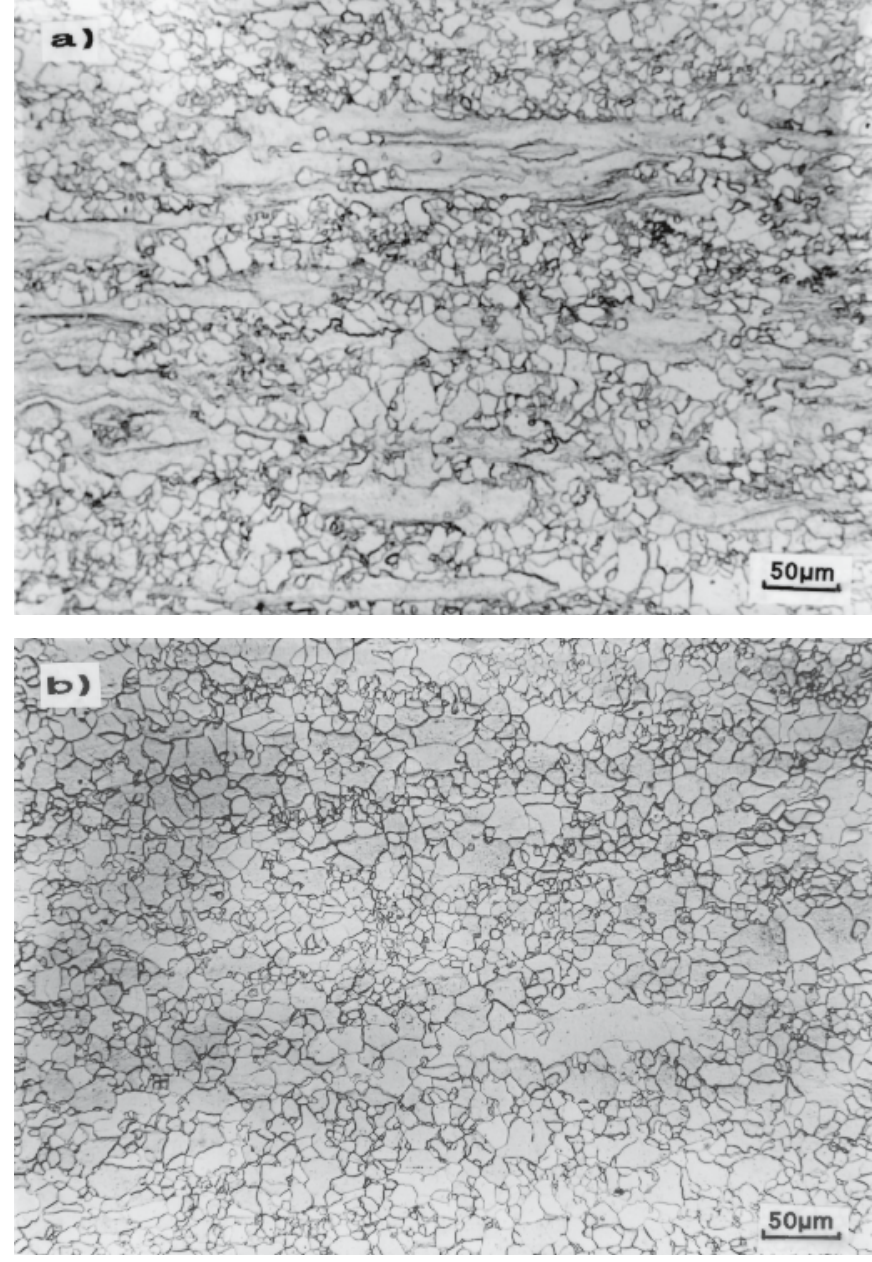

Figura 3. Microestructuras obtenidas en el plano de laminación del acero recocido a las temperaturas: a) $600^{\circ} \mathrm{C}$ y b) $620^{\circ} \mathrm{C}$

$7 \mathrm{AR}$

$\& E F \&$

13.83

$\begin{array}{lllll}.70 & 1.00 & 1.40 & 2.00 & 2.80\end{array}$

$\begin{array}{lllll}4.00 & 5.60 & 8.00 & 11.0 & 16.0\end{array}$

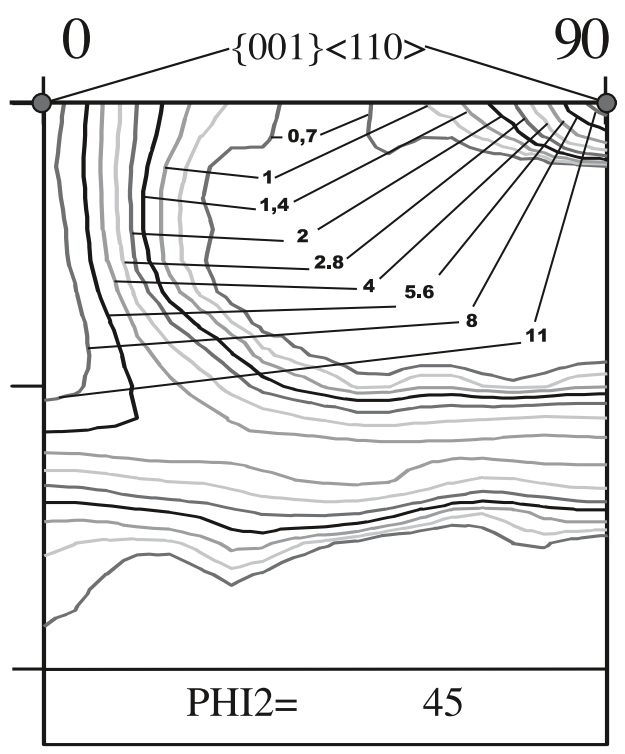

Figura 4. Sección $\varphi_{2}=45$ de la FDO del presente acero en las condiciones de laminación en frío. 
taciones dadas por diferentes autores (5) en las que se proponen que los primeros granos deformados que son consumidos al inicio de la recristalización pertenecen a la Fibra $\gamma$, mientras que aquellos que persisten en el estado deformado tienen orientaciones cercanas a la Fibra $\alpha$. La razón de una mayor rapidez en la nucleación asociada a las orientaciones $\{111\}$ va ligada a un mayor nivel de la energía almacenada en estas zonas durante la deformación en frío (6). Estos comportamientos corresponden a un mecanismo de nucleación orientada. Adicionalmente, los primeros granos originados al comienzo de la recristalización mantienen la misma orientación que los de la estructura deformada en frío de donde proceden (7). Esto explica que los primeros estadios de la recristalización no ocasionen un cambio aparente en la intensidad de las dos fibras, $\gamma$ y $\alpha$.

$\mathrm{Al}$ subir la temperatura del ciclo de recocido se produce un importante fortalecimiento de la Fibra $\gamma$ y un debilitamiento de la $\alpha$. A partir de la temperatura de $620^{\circ} \mathrm{C}$, que corresponde a un $86 \%$ de recristalización, ya se percibe la intensificación de toda la Fibra $\gamma$, apareciendo un pico de mayor intensidad en la orientación (111)[1112]. Paralelamente, en la Fibra $\alpha$ hay una fuerte disminución en las densidades de las componentes comprendidas entre (001) [110] y (112) [11̄0], encontrándose dos picos de máxima intensidad en las orientaciones (114) [11 0] y (111) [110]. A temperaturas superiores, en las que el acero se encuentra completamente recristalizado, antes de que concluya el ciclo de recocido y por tanto entre en la etapa de crecimiento del tamaño de grano, continua el fortalecimiento de la Fibra $\gamma$ acompañado por una disminución de las componentes comprendidas entre (001) [11̄0] y (112) [110] de la Fibra $\alpha$.

Las orientaciones (112) [110] y (111)[1̄12], involucradas en estos cambios, están relacionadas cristalográficamente por un giro de $35^{\circ}$ alrededor de un eje común $<110>$. Esta situación está cerca de la condición de máxima velocidad de crecimiento de los nuevos granos recristalizados en la matriz deformada, que para el caso de los materiales con estructura cúbica centrada en el cuerpo, como la ferrita, se ha determinado que se verifica para juntas de grano que pueden describirse mediante rotación de $27^{\circ}$ alrededor de $<110>$ (8). La disminución observada en la componente (112) [110], al mismo tiempo que se produce el aumento de la componente (111) [1112] parece indicar una importante participación del crecimiento selectivo en la formación de la textura de recristalización en los últimos estadios de esta última. Resultados similares se pueden encontrar en trabajos anteriores $(9,10)$.

Ambos mecanismos, el de nucleación orientada que favorece la formación de granos con orientación $\{111\}$ durante la recristalización y el de crecimiento selectivo posterior, contribuyen a la formación de una textura favorable para la embutición. Sin embargo, la optimización de esta última basándose en la obtención de valores del coeficiente de Lankford $\left(\mathrm{r}_{\mathrm{m}}\right)$ elevados unidos a una importante isotropía planar (11), valores de $\Delta r_{m}$ bajos, implican un intenso control del tamaño de grano y de los niveles de nitrógeno libre en la banda en caliente (12). Por otro lado, es imprescindible, la determinación de la combinación adecuada de reducción en frío y condiciones de recocido si se quiera alcanzar la citada optimización de la textura de embutición (13).

En último lugar, para las temperaturas de recocido más elevadas, comprendidas en el rango $740-800^{\circ} \mathrm{C}$, se ha observado que se produce un debilitamiento de la textura. En la Fig. 7, se puede observar la disminución en la densidad de la Fibra $\gamma$ que, a pesar de ello, sigue manteniendo el mismo perfil con

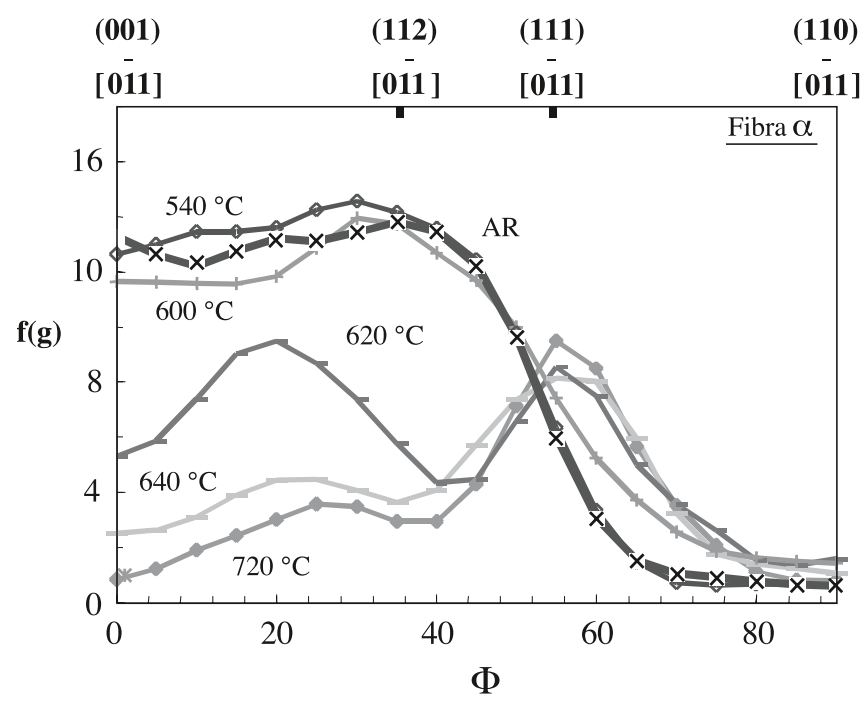

Figura 5. Densidad de orientaciones, $\mathrm{f}(\mathrm{g})$, a lo largo de la Fibra $\alpha$ para diferentes temperaturas de recocido.

(111)

(111)

[011]

[112]

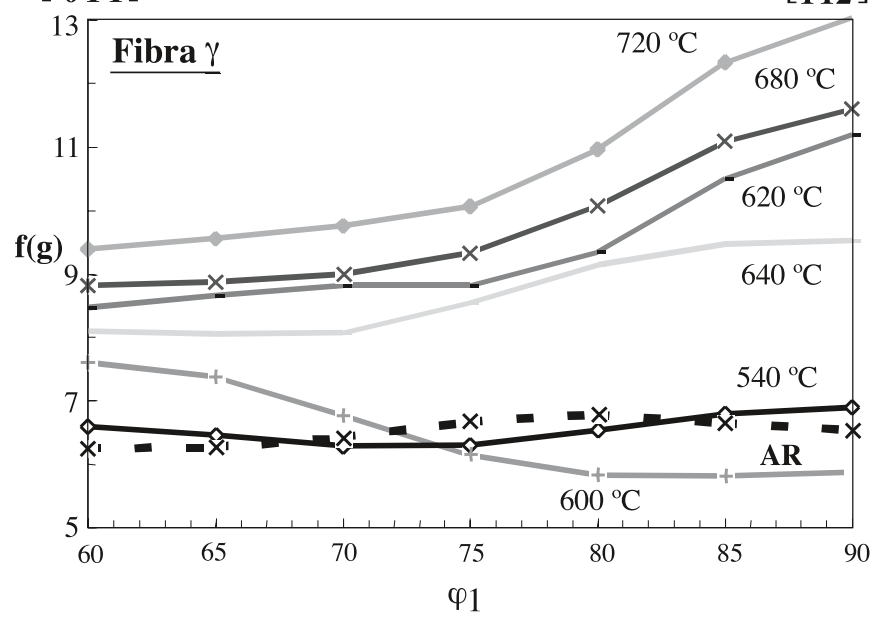

Figura 6. Densidad de orientaciones, $\mathrm{f}(\mathrm{g})$, a lo largo de la Fibra $\gamma$ para diferentes temperaturas de recocido.

(111)

[0 1111$]$

(111)

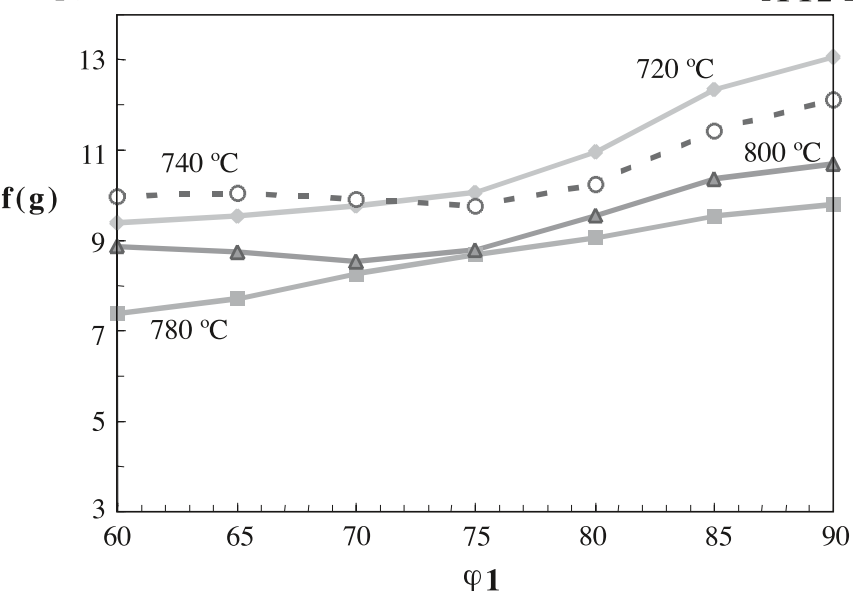

Figura 7. Densidad de orientaciones, $\mathrm{f}(\mathrm{g})$, a lo largo de la Fibra $\gamma$ para altas temperaturas de recocido. 
un pico de intensidad en la orientación (111)[1̄112]. Este debilitamiento de la Fibra $\gamma$ ya ha sido comentado por varios autores $(10,14,15,16)$ y está relacionado con los cambios involucrados en la transformación parcial a austenita que tiene lugar a temperaturas superiores a $\mathrm{A}_{1}$. Este deterioro de la textura puede minimizarse mediante el control de la transformación inversa a ferrita que tiene lugar durante la etapa de enfriamiento en el ciclo de recocido continuo (14)

\section{CONCLUSIONES}

El progreso de las texturas experimentado por un acero ultrabajo en carbono y calmado con aluminio, al variar la temperatura de recocido, puede ser explicado mediante una combinación de los mecanismos de nucleación orientada y de crecimiento selectivo.

Durante la primera mitad de la recristalización la nucleación tiene lugar en granos deformados con orientaciones próximas a las de las componentes de la Fibra $\gamma$, mientras que la Fibra $\alpha$ no se ve afectada. Este comportamiento se asocia a una nucleación orientada de los nuevos granos de ferrita que se forman en la recristalización.

La mejora de la textura que supone el fortalecimiento de la Fibra $\gamma$ coincide posiblemente con un importante crecimiento selectivo de grano que puede iniciarse antes de que concluya totalmente la recristalización.

\section{BIBLIOGRAFÍA}

1. R. K. Ray, J.J. Jonas, R. E.. HooK. Cold rolling and annealing textures in low carbon and extra low carbon steels. Inter. Mater. Rev., 39, 129-172 (1994).

2. I. Gutiérrez, M.M. Petite, J.I. Larburu, J. Zaitegui, W. B. Hutchinson, D. Artymowicz, P. J. Evans, G. J. Spurr, H. K. D. H. Bhadeshia y N. Chester. "Modelling of microestructural development during continuous annealing process". Proyecto CECA 7210.EC/808, 940, 941,942. Informe Final. Año 1998.
3. P. Van Houtte, The MTM-FHM Software System Version 2, Users Manual, 1995.

4. H. Shi, M. Atkinson y D. Dunne. Ultra-rapid annealing of cold rolled iron by resistance heating. Recrystalisation 90, Ed. T. Chandra., The Minerals, Metals and Materials Society, 735-740 (1990).

5. I. L. Dillamore, W. B. Hutchinson. The control of grain size and orientation in sheet steel. Trans. ISIJ, 11, 877-883 (1974).

6. I.L. Dillamore, C. J. E. Smith y T. W. Watson. Oriented nucleation in the formation of annealing textures in iron. Met. Sci. Journal, 1, 49-54 (1967).

7. B. Plutka y H. P. Hougardy. Recent results on recrystallization mechanism in deep drawing steels achieved by single orientation measurementes. Recrystallizarion'92, Ed. M. Fuentes y J. Gil Sevillano, Mat. Sci. Forum, 113115, 183-188 (1993).

8. G. Ibe y K. Lücke. Correlations of orientation during the recrystallization of single crystals of an iron-silicon alloy containing $3 \% \mathrm{Si}$. Arch. Eisenhüttenwes, 39, 693-703 (1968).

9. F. Emren, U. V. Schlippenbach y K. Lücke. Investigation of the development of the recrystallization textures in deep drawing steels by ODF analysis. Acta Metall, 34, 2105-2117 (1986).

10. X. C. Mi y B. Y. Kong. Effect of annealing on recrystallization texture in IF steel. $11^{\text {th }}$ Int. Conf. on "Textures of Materials", ICOTOM 11, China, Ed. Z. Liang y col., International Academic Publishers, 824-829 (1996).

11. H. P. Stüwe y J. Faustmann. Introducción a las texturas de los materiales metálicos. Ed. Montecorvo, Madrid (1969).

12. M. M. Petite. Tesis doctoral, ESII de San Sebastián, Universidad de Navarra (1999).

13. J. C. Herman, L. Kestens, I. Gutiérrez, J.L. Bocos, J. Zaitegui, V. Cascioli, P.E. Di Nunzio y R. Grobterlinden. “ Texture control in cold rolled steel sheets for an optimized anisotropy". Proyecto CECA n ${ }^{\circ}$ 7210-EC/945. Informe técnico. Año 1998.

14. R. Horta , D. W. Wilson y W. T. Roberts. Texture control in the rapid annealing of lowcarbon steels. Journal of The Iron and steel Institute, 210, 42-51 (1972).

15. S. Ono , P. Deleneau y B. Thomas. The influence of intercritical annealing temperatures and times in simulated conditions of continuous annealing on the drawability of Al-Killed low carbon steels. Role of initial carbide morphologies. $7^{\text {th }}$ Int. Conf. on "Textures of Materials". ICOTOM 7, Netherlands, Ed. C. M. Brakman y col., Society for Materials Sience, 359-365 (1984).

16. F. Emren y K. Lücke. On the textural development of rapidly annealed deep drawing steels. $7^{\text {th }}$ Int. Conf. on "Textures of Materials", ICOTOM 7, Netherlands, Ed. C. M. Brakman y col., Society for Materials Sience, 397-402 (1984). 